\title{
Dual processes in recognition and in recognition failure
}

\author{
J. RICHARD HANLEY \\ Newcastle Polytechnic, Newcastle upon Tyne, England
}

\begin{abstract}
It is argued that adherence of the results of experiments in the recognition failure paradigm to the function observed by Tulving and Wiseman (1975) reflects the operation of two distinct processes in recognition similar to those described by Mandler (1979, 1980). Familiarity decisions based on the target word alone can occur when target and list cue have not been integrated and result in some measure of dependence between recall and recognition. Contextual retrieval can operate in recognition when integration has occurred, and is independent of success in recall. It is further contended that deviations from the Tulving-Wiseman function arise because special instructions given to subjects produce more or less cue-target integration than is normal, and differentially affect the relative proportion of items recognized via contextual retrieval and via familiarity decisions. The first part of the paper argues that the contextual retrieval involved in recognition failure experiments takes the form of an attempted backward recall of the list cue from the target, consistent with the suggestions of Rabinowitz, Mandler, and Barsalou (1977) and claims that the results of a paper by Fisher (1979) strongly support this view.
\end{abstract}

When target words have been learned in the presence of specific list cues, subjects are frequently able to recall items that they had previously been unable to recognize. The recognition failure of recallable words, as this finding has come to be known, generally occurs when recognition is tested in the absence of list cues and recall is tested by asking subjects to remember the targets in the presence of the original cues (Tulving \& Thomson, 1973; Watkins \& Tulving, 1975). The phenomenon of recognition failure presents a major challenge for theories that attempt to account for the relationship between recognition and recall. In 1978, however, Flexser and Tulving put forward a model that was able to explain not only the existence of recognition failure, but also the reason why the proportion of recallable words that fail to be recognized in any given experiment can usually be accurately predicted from the overall recognition hit rate (Tulving \& Wiseman, 1975).

The purpose of this theoretical note is to suggest that the views of George Mandler and his colleagues (Mandler, 1979, 1980; Rabinowitz, Mandler, \& Barsalou, 1977) provide the basis for an alternative explanation of recognition failure to that proposed by Flexser and Tulving (1978). It is argued that this new explanation can also account for the phenomenon of recognition failure and the relationship between recognition and recall described by Tulving and Wiseman (1975). In addition, unlike the model of Flexser and Tulving, the present account can

I would like to thank Bryan Watson, Janice Hindhaugh, Marie Scholes, and, in particular, the reviewers of this and previous versions of the manuscript for having brought to my attention several of the points raised in the article. Correspondence should be addressed to J. R. Hanley, School of Behavioural Science, Newcastle Polytechnic, Newcastle Upon Tyne NE1 8ST, England. accommodate the deviations from that function observed by Begg (1979), Fisher (1979, Experiment 2), and Gardiner and Tulving (1980).

One of Mandler's most important assumptions is that two distinct processes are involved in recognition. One of these is based on the perceptual familiarity of the target item, while the other involves contextual retrieval. Rabinowitz, Mandler, and Barsalou (1977) believe that recognition failure results from a failure of contextual retrieval-implicit backward recall of the list cue-in situations in which ordinary forward recall is successful. The first part of this article will contrast this concept of "retrieval asymmetry" with the somewhat different proposal of Flexser and Tulving (1978). It will be argued that strong support for Rabinowitz, Mandler, and Barsalou's views derives from the results of Fisher (1979, Experiment 1). In the second part of the article, some major advantages of adopting Rabinowitz, Mandler, and Barsalou's position should become apparent. It is argued that successful backward recall is normally required for the recognition of most, but not all, of the $B$ items in a list of A-B pairs. For those that have not been integrated or "unitized," recognition will depend on the other process suggested by Mandler and his colleagues-a familiarity decision on the B item itself. It is concluded that adherence to, and deviations from, the TulvingWiseman function can be seen in terms of a probabilistic mix of items recognized by familiarity decisions and via contextual retrieval.

\section{RECOGNITION FAILURE AND RETRIEVAL ASYMMETRY}

As mentioned above, Rabinowitz, Mandler, and Barsalou (1977) attempt to explain recognition failure in terms 
of retrieval asymmetry. That is, recognition failure occurs when the $A$ item elicits the $B$ item in recall, but when at recognition the $B$ item fails the familiarity test and also fails to provide access to the A item implicitly in backward recall. Recall superiority over recognition (see Wiseman \& Tulving, 1976) would be expected whenever there is a general tendency at retrieval for cues to elicit targets more easily in recall than targets implicitly elicit list cues during recognition. The support for this position comes from Rabinowitz et al.'s final four experiments. They found that when the target successfully elicited the cue in a surprise backward recall test, there was very low incidence of recognition failure. Overall, there was a strong positive correlation between recognition failure and situations in which the list cue elicited the target in forward recall and the target failed to elicit the cue in backward recall.

Unfortunately, though, none of the evidence provided by Rabinowitz, Mandler, and Barsalou (1977) is, in fact, particularly compelling. The low recognition failure levels observed when backward recall was successful were accompanied by high overall recognition hit rates. In such a situation, one would expect low recognition failure simply on the basis of the Tulving-Wiseman function, and therefore the small observed incidence of recognition failure might not have been the direct result of the successful backward recall. The correlation between retrieval asymmetry and recognition failure is equally ambiguous. As Flexser and Tulving have pointed out,

It is difficult to establish the casual connection between recognition failure and backward recall. Recall of A may fail because B is not recognized or B may fail to be recognized because it does not (implicitly) retrieve A. Rabinowitz et al. (1977) favor the latter alternative, although neither their data nor any other data we know of discriminate between the alternatives. (Flexser \& Tulving, 1978, p. 157).

Rabinowitz, Mandler, and Barsalou are arguing, therefore, that recognition may fail because the $B$ item does not cue the $A$ item. It is impossible, however, to choose between that interpretation and one that suggests that the $B$ item does not successfully access the episodic memory trace for the encoded event and, because of this, the A item cannot be retrieved. However, there are some results, namely those of Fisher (1979, Experiment 1), that provide rather better evidence that this hypothetical backward recall strategy actually takes place during recognition than is provided by Rabinowitz, Mandler, and Barsalou themselves.

Fisher (1979) performed two experiments designed to investigate the relationship between recognition and recall. As in many previous experiments, A-B word-pairs were used as stimulus materials, with recall tested in the presence of list cues and recognition tested in their absence. Of particular interest was the fact that Fisher manipulated the degree of association between the $\mathrm{A}$ items and $B$ items. His four conditions comprised one in which there was a relatively high amount of preexperimental association between the two items and another in which there was relatively low association between the items. In the remaining two conditions, there was a preexperimental asymmetry in the association between the pairs. That is, in one condition, there was a high association going from the $A$ item to the $B$ item but a low association from the $B$ item to the $A$ item. In the final condition, there was high association from the $B$ item to the $A$ item but low association from the $A$ item to the $B$ item.

One of Fisher's most interesting results was that recall benefited from a strong preexperimental association going from cue to target relative to the no-association condition but was unaffected by any strong association going from target to cue. For recognition, however, the opposite was true. Recognition was not improved by a strong cue-to-target relationship but benefited from a strong association from target to cue relative to the no-association condition. Hanley and Hindhaugh (1982) replicated these results in a typical recognition failure experiment in which both adjectives and nouns were used as targets and also as cues. Fisher $(1979$, p. 231) argues that his results are consistent with the positions of both Flexser and Tulving (1978) and Rabinowitz, Mandler, and Patterson (1977) in that "in both recall and recognition, it was expected and found that the ease of accessing the encoded event from the retrieval cue was an accurate predictor of performance." However, although Fisher's results might not necessarily be incompatible with the model of Flexser and Tulving, they appear to be much more easily explained by the theory of Rabinowitz, Mandler, and Barsalou (1977).

Flexser and Tulving (1978) argue that retrieval is likely to be successful to the extent that the retrieval information in the cue matches the episodic trace sufficiently closely. An adherent of their position could, after examining Fisher's data, argue that when the strong association went from cue to target, then the similarity between cue and episodic trace was in general greater than the similarity between target and episodic trace, and that is why recall was superior to recognition. Similarly, it could be argued that when the strong association went from target to cue, then, in that situation, the similarity between the cue and episodic trace was smaller than the similarity between the target and the episodic trace, and hence recognition was superior to recall. Although there is no obvious reason why this should be the case, nonetheless it cannot be claimed that the results are incompatible with Flexser and Tulving's views, then. The finding that recognition benefits from a strong preexperimental association going from target to cue, however, is exactly what one would expect on the basis of the theory of Rabinowitz, Mandler, and Barsalou. From their perspective, the superior recognition performance would reflect the greater accessibility of the list cue during backward recall. The theory of Rabinowitz, Mandler, and Barsalou (1977) appears, then, to provide a convincing explanation of why certain pairs of words exhibit a higher probability of recognition failure than others. At the very least, it now seems that, in light of Fisher's results, it would be premature to dismiss retrieval asymmetry as a possible explanation of recog- 
nition failure on the basis of the criticisms made by Flexser and Tulving (1978) about its applicability. ${ }^{1}$

On the other hand, Flexser and Tulving's model was designed to explain the Tulving-Wiseman function, the observation that level of recognition failure is predictable from the overall recognition hit rate but is unaffected by level of recall (Tulving \& Wiseman, 1975). The theory of Rabinowitz, Mandler, and Barsalou has not been specifically applied to this issue, however. It is therefore important that a comparison of the two theoretical positions should attempt to investigate whether an explanation incorporating the concept of retrieval asymmetry can also be used to explain the Tulving-Wiseman function.

\section{THE TULVING-WISEMAN FUNCTION}

Having examined the results of a wide range of experiments from several laboratories that used the recognition failure paradigm, Tulving and Wiseman (1975) observed a highly systematic relationship between $\mathrm{p}(\mathrm{rgn})$, recognition hit rate, and $\mathrm{p}(\mathrm{rgn} / \mathrm{rcl})$, the proportion of recallable words that could be recognized. $\mathrm{P}(\mathrm{rgn} / \mathrm{rcl})$ is therefore recognition conditionalized on successful recall, and recognition failure is defined as the complement of this term. The Tulving-Wiseman function formally specifies the relationship between the two measures as follows: $\mathrm{p}(\mathrm{rgn} / \mathrm{rcl})=\mathrm{p}(\mathrm{rgn})+.5 \mathrm{p}(\mathrm{rgn})-\mathrm{p}(\mathrm{rgn})^{2}$. From the point of view of generate-recognize theories of recall and recognition (Kintsch, 1970), recognition is seen as one of two stages involved in recall and must always be successful if recall is successful. The proportion of recallable words recognized should therefore be 1 and recognition failure 0 . If, on the other hand, recall and recognition are quite independent of one another-in other words, successful recall says nothing about whether or not recognition will be successful-then the proportion of recallable words that can be recognized should be equal to the overall recognition hit rate. In fact, Tulving and Wiseman showed that the data fell somewhere between these two extremes. However, the proportion of recallable words recognized was consistently only slightly higher than the recognition hit rate, clearly contradicting the Kintsch (1970) model and suggesting that there was a considerable degree of independence between recall and recognition.

According to Flexser and Tulving (1978), the trend toward independence between recall and recognition comes about because the retrieval information in the list cue is uncorrelated with the retrieval information contained in the copy cue. However, both types of cue are aimed at the same memory trace, which will vary in terms of how many encoded features it includes. Such variability in the "goodness of encoding" will affect the probability that either type of cue will recreate the episodic memory trace. This produces the small degree of dependence between recognition and recall observed by Tulving and Wiseman (1975).

The dual-process views of Mandler $(1979,1980)$ and Rabinowitz, Mandler, and Barsalou (1977), however, pro- vide the basis for a rather different account. Their position is that on some occasions items can be recognized on the basis of a familiarity decision on the target item itself, whereas for others the retrieval of the encoding context is necessary. In what circumstances are either of these two types of process likely to be more or less successful than the other? Mandler (1979, p. 307) suggests that in the paired-associate paradigm the $\mathrm{A}$ item and the $\mathrm{B}$ item tend to be stored "holistically" in memory "as a unit structured by the relational coding of the two words." Crowder (1976, pp. 408-409) argued similarly that "when items enter into such a cohesive gestalt they lose their individual identity." The literature on paired-associate learning provides considerable support for such a position (e.g., Horowitz \& Prytulak, 1969; Roediger \& Adelson, 1980). Let us assume, therefore, that in these circumstances the familiarity decision on the $B$ item alone is less likely to prove successful because it is not the B item that is familiar-it is the A-B pair that is familiar. Thus, before either recall or recognition can occur, the subject must first retrieve the entire A-B unit. In other words, these are occasions when a positive decision cannot be made solely on the basis of the retrieval information provided by the experimenter. The correct decision will be made only if the subject manages to implicitly retrieve the missing member of the A-B pair (by forward recall during cued recall or by backward recall during recognition) and then uses the entire A-B unit to access the trace in episodic memory. This sort of process in which a subject actively searches memory for additional retrieval information is similar to what Baddeley (1982, p. 712) refers to as "recollection."

What, though, of the relationship between the probability that the A item will elicit the B item in forward recall and the probability that the $B$ item will access the $A$ item in backward recall? It will be remembered from the previous section that Fisher (1979, Experiment 1) showed that recall was unaffected by the target-to-cue association, although it was greatly improved by a strong cue-to-target association. Conversely, recognition did not improve when there was a strong cue-to-target association but did benefit from a strong target-to-cue association. This seems to indicate that the ability of the target to elicit the cue, although crucial for recognition, has no effect on cued recall, whereas the ability of the cue to elicit the target, although crucial for recall, has no effect on recognition. Fisher's finding is exactly what one would predict if the relatively low correlation between successful recall and successful recognition observed by Tulving and Wiseman (1975) comes about because there is no correlation between success on forward recall and success on backward recall. This, then, constitutes an alternative account of what Flexser and Tulving (1978) refer to as "retrieval independence."

Such, anyway, is likely to be the consequence of an elaborate encoding, in which the subject has succeeded in integrating cue and target during encoding. Let us assume, though, that while learning this sort of list a subject does not have the desire, sufficient ingenuity, or 
available time to integrate all the pairs and for some pairs instead simply rote rehearses the target word. ${ }^{2}$ In such a situation, integration of cue and target is improbable. Nonetheless, the familiarity of the target word itself should be increased, and thus a successful decision on the basis of the $\mathrm{B}$ item alone becomes the way in which it is most likely to be recognized. In light of this, there will probably always be a few items in any list for which the question of whether the target provides access to the list cue during recognition is irrelevant. Backward recall should not play any functional role in recognition, then, when a decision has to be made on the $B$ item itself rather than on the A-B pair. Although one would not expect target words encoded in such a way to be particularly well remembered, their recognition would not depend on any contextual retrieval. Therefore, if subjects manage to recall any of these items, they should also be able to recognize them.

The suggestion, therefore, is that there are two distinct processes that can occur in both recall and recognition. Eventually, a decision must be made on the basis of the available retrieval information. Since this decision process is assumed to be equivalent in both types of test, it is seen as a cause of dependence between recognition and recall. When recognition requires no contextual retrieval, and a decision can be made on the basis of the target word itself, then there should be overall dependence between recall and recognition-if a word can be recalled, it should also be recognized. In this situation, the model of Kintsch (1970) is probably correct in that recognition can be usefully characterized as requiring only one of the two processes involved in recall. When, however, the target and cue have become unitized, then the decision must be made on the basis of the A-B pair rather than on the $B$ item alone. Successful recognition will not occur unless the subject is first able to supplement the retrieval information provided by the experimenter by accessing the list cue. The argument is that the strong trend toward independence between recall and recognition found in the recognition failure paradigm comes about because the ability of the target to provide this extra information is uncorrelated with the ability of the cue to access the target. This trend is to some extent countered by the existence of a common decision process operating in both recall and recognition, which means that there may be at least some dependence between recall and recognition. This relationship will be much stronger, however, if there are any target items recognized by a familiarity decision on the B item rather than following contextual retrieval. The presence of only a few items recognized in this way would result in recallable words being recognized appreciably better than nonrecallable words. The existence of such items may be the primary reason why the line of best fit to the data on the graph on page 155 of Flexser and Tulving's (1978) article, which plots the relationship between overall recognition level and the recognition level of recallable words, is slightly above the diagonal line that would indicate complete stochastic independence between recall and recognition. This, then, explains the degree of dependence observed between recall and recognition by Tulving and Wiseman and constitutes an alternative to Flexser and Tulving's "goodness-of-encoding principle" and Begg's (1979) "vandal."

It might be noted that this explanation of the adherence of the recognition failure data to the Tulving-Wiseman function bears some similarity to a proposal made by Jones (1978). He also argues that these results reflect the outcome of two kinds of processes, although the processes suggested by Jones-extrinsic and intrinsic knowledge in recall-are quite different from the processes outlined above. The reason why the present account appears preferable is, it will be argued below, that it provides a framework in which deviations from the Tulving-Wiseman function can also be explained. These data pose a problem for the Flexser and Tulving (1978) model, which, as Gardiner and Tulving (1980) admit, contains no obvious element with which to explain the deviations. In addition, none of the alternative explanations of the TulvingWiseman function that Gardiner and Tulving consider, including that of Jones (1978), fare any better with these data.

\section{DEVIATIONS FROM THE TULVING-WISEMAN FUNCTION}

Gardiner and Tulving (1980) found that for certain types of items the data did not fit the Tulving-Wiseman function. When the $A$ item was a two-digit number, and also when both $A$ and $B$ items were abstract words, then there was a much stronger than usual probability that recallable words would also be recognized. One difference between these kinds of pairs and those used in previous studies is that the members of these pairs might be difficult to relate together during encoding. Consistent with this, when subjects were given specific instructions on how to integrate the items, then the data points were much closer to the Tulving-Wiseman function. When pairs of items are difficult to integrate, a holistic encoding of A and B items is not likely to have been achieved and the retrieval and decision processes are likely to be different from normal circumstances. Specifically, a familiarity decision based on one member of a pair has more chance of being successful relative to a decision on the intact A-B pair than is the case when the pairs have been successfully integrated. It was argued earlier that the presence of only a few target items recognized by a familiarity decision on the $B$ item will increase the dependence between recall and recognition. In Gardiner and Tulving's study, an abnormally high proportion of recognition hits are likely to have come about through a familiarity decision on the $B$ item rather than following contextual retrieval. It would therefore follow that there should also be an unusually high degree of dependence between recall and recognition in their study. In such a situation, virtually all words that can be recalled should also be recognized-hence the deviations from the 
Tulving-Wiseman function that Gardiner and Tulving (1980) observed.

The other recorded situation in which deviations from the function were observed is also connected with the type of encoding subjects were asked to carry out. Begg (1979, Experiment 1) and Fisher (1979, Experiment 2) asked some subjects to learn pairs of words by repeating them (maintenance rehearsal) and asked other subjects to learn by creating a meaningful link between the two words (elaborative rehearsal). If one examines the data as a whole, then the relationship between recognition and the recognition level of recallable words closely fits the Tulving-Wiseman function. When the data from the elaborative and maintenance conditions are analyzed separately, however, then in the elaborative condition a lower proportion of recallable words were recognized than would be predicted and in the maintenance condition a higher proportion of recallable words were recognized than would be predicted.

These results, consistent with Bahrick's (1979) suggestion that, when organization has occurred, then recall and recognition are considerably less dependent on each other than is the case with no organization, can be explained in a similar way to those of Gardiner and Tulving (1980). Elaborative rehearsal is likely to establish strong links between an item and its encoding context, whereas maintenance rehearsal appears to involve relatively little cuetarget integration (Mandler, 1979; Nairne, 1983). Consequently, for the reasons presented earlier, maintenance rehearsal will lead to a higher proportion of recallable items being recognized than will a more elaborate encoding. Wiseman function represents recognition of a probabilistic mix of integrated and nonintegrated items. This means that, for some items, recall and recognition are dependent, whereas for others, they are much less closely correlated. The consequence of this is that, for ordinary lists, under normal instructions, the proportion of recallable items recognized is slightly above the line of stochastic independence. Exceptions occur when there is a preponderance of nonintegrated pairs (Begg, 1979; Fisher, 1979; Gardiner \& Tulving, 1980) and recognition failure is lower than predicted, or there is a greater than usual preponderance of integrated pairs (Begg, 1979; Fisher, 1979) when recognition failure will be higher than predicted.

\section{CONCLUSION}

The main point of this article is to suggest that an account based on the views of Mandler, Rabinowitz, and their associates can be shown to provide a framework within which recognition failure can be clearly understood. First, it was argued that the results of Fisher (1979) suggest that the ability of the target to elicit the cue is of vital importance in recognition, rather than being merely a consequence of recognition. Second, it was suggested that adherence to and deviations from the TulvingWiseman function can be seen in terms of a probabilistic mix of two types of processes-familiarity decisions on the $\mathrm{B}$ item alone and decisions that follow contextual retrieval. This ability to explain deviations from the Tulving-Wiseman function seems to give the present account an advantage over Flexser and Tulving (1978).

Because it is assumed that dependency between recall and recognition reflects the operation of an identical decision process in both recognition and recall, the theory in some respects resembles earlier two-stage models such as that put forward by Kintsch (1970). The Achilles' heel of Kintsch's model turned out to be its inability to accommodate the fact that context effects frequently lead to a breakdown in this relationship, and words are successfully recalled without being recognized. According to the present account, recognition failure becomes possible when a target word has become integrated with its encoding context and recognition initially requires some form of contextual retrieval. Since this will be a form of retrieval significantly different from that which is typically involved in cued recall, success in recall and recognition will no longer be closely correlated. Hence, as the conditions of an experiment make it more likely that unitization of cue and target will occur, so the proportion of recallable words recognized will correspond more closely to the overall level of recognition. The present account differs from the encoding specificity principle by maintaining that a target word does not automatically become integrated with its encoding context. Only when unitization has occurred is performance crucially affected by the presence or the accessibility of the original list cue while the recognition decision is being made.

\section{REFERENCES}

BAdDeley, A. D. (1982). Domains of recollection. Psychological Review, 89. 708-729.

BAHRICK, H. P. (1979). Broader methods and narrower theories for memory research. In L. S. Cermak \& F.I.M. Craik (Eds.), Levels of processing in memory. Hillsdale, NJ: Erlbaum.

BEGG, I. (1979). Trace loss and the recognition failure of unrecalled words. Memory \& Cognition, 7, 113-123.

CROWDER, R. G. (1976). Principles of learning and memory. Hillsdale, NJ: Erlbaum.

FisHER, R. P. (1979). Retrieval operations in cued recall and recognition. Memory \& Cognition, 7, 224-231.

FLEXSER, A. J., \& TulviNG, E. (1978). Retrieval independence in recognition and recall. Psychological Review, 85, 153-171.

GARDINER, J. M., \& TUlVING, E. (1980). Exceptions to recognition failure of recallable words. Journal of Verbal Learning and Verbal Behavior, 19, 194-209.

HaNley, J. R., \& Hindhaugh, J. (1982, December). The relationship between retrieval asymmetry and recognition failure. Paper presented at the British Psychological Society London Conference, University of London Institute of Education, London, England.

Horowitz, L. M., \& PrytulaK, L. S. (1969). Redintegrative memory. Psychological Review, 76, 519-531.

JONEs, G. V. (1978). Recognition failure and dual mechanisms in recall. Psychological Review, 85, 464-469.

KINTSCH, W. (1970). Models for free recall and recognition. In D. A. Norman (Ed.), Models of human memory. New York: Academic Press.

MANDLER, G. (1979). Organization and repetition: An extension of organizational principles with special reference to rote learning. In $\mathbf{L}$. $\mathbf{G}$. Nilsson (Ed.), Perspectives on memory research. Hillsdale, NJ: Erlbaum. 
MandleR, G. (1980). Recognizing: The judgment of previous occurrence. Psychological Review, 98, 252-271.

NAIRNE, J. S. (1983). Associative processing during rote rehearsal. Journal of Experimental Psychology: Learning, Memory, and Cognition, 9, 3-20.

Rabinowitz, J. C., Mandler, G., \& Barsalou, L. W. (1977). Recognition failure: Another case of retrieval failure. Journal of Verbal Learning and Verbal Behavior, 16, 639-663.

Rabinowitz, J. C., Mandler, G., \& Patterson, K. E. (1977). Determinants of recognition and recall: Accessibility and generation. Journal of Experimental Psychology: General, 106, 302-329.

Roediger, H. L., \& ADELSON, B. (1980). Semantic specificity in cued recall. Memory \& Cognition, 8, 65-74.

Tulving, E., \& Thomson, D. M. (1973). Encoding specificity and retrieval processes in episodic memory. Psychological Review, 80, 352-373.

Tulving, E., \& Wiseman, S. (1975). Relation between recognition and recognition failure of recallable words. Bulletin of the Psychonomic Society, 6, 79-82.

WatKINS, M. J., \& Tulving, E. (1975). Episodic memory: When recognition fails. Journal of Experimental Psychology: General, 103, 5-29.

Wiseman, S., \& Tulving, E., (1976). Encoding specificity: Relation between recall superiority and recognition failure. Joumal of Experimental Psychology: Human Learning and Memory, 2, 349-361.

\section{NOTES}

1. At first sight, it might seem reasonable to object to the present explanation of recognition failure by citing the established finding that recognition failure occurs even when the preexperimental association between the members of each pair is symmetrical (e.g., Wiseman \& Tulving, 1976). This result does not contradict the above account, however, even if one were to attribute probability of recall solely to the $A \rightarrow B$ association and probability of recognition solely to the $B \rightarrow A$ association. The argument is, first, that recognition failure occurs when in recall the cue elicits the target at testing but in recognition the target fails to elicit the cue and, second, that neither of these two outcomes is dependent on the other. Recognition failure does not occur only when the probability of the target eliciting the cue is smaller than the probability that the cue will elicit the target. For instance, if there was a symmetrical preexperimental association of .5 between the members of each pair in a list and this miraculously exactly reflected the chance that the cues and the targets would elicit one another at testing, then one would clearly expect recognition failure to occur. For half the pairs in which the cue successfully accessed the target at recall, the target would fail to elicit the cue at recognition. This would result in recognition failure for these items (on the assumption that the familiarity decision on the B item fails also). Recall superiority, however, would not be expected without there being a stronger preexperimental association going from cue to target than from target to cue.

2. Even if the subject were to rote rehearse both members of a pair together, unitization would be unlikely to occur, in light of the evidence that time spent performing maintenance rehearsal does not integrate an item with its encoding context (e.g., Nairne, 1983). Nonetheless, it might be assumed that if unitization of the A and B items has not occurred, then the A item cannot possibly retrieve the B item and the proportion of these target items that can be recalled should be zero. However, it is quite conceivable that under such circumstances the $A$ item could still cue the B item-in the same way that an extralist cue could elicit the $B$ item, perhaps. Although such a view may not be consistent with the encoding specificity principle, Baddeley (1982) makes a similar sort of distinction between "independent" and "interactive" context.

(Manuscript received June 28, 1983; revision accepted for publication July 5, 1984.) 